\title{
Determinación de los Parámetros del Modelo de Flory- Huggins para estimación del Punto de Nube de Surfactantes No lónicos
}

\author{
Alessandro Alisson de L. Araújo ${ }^{(1) \star}$ Eduardo L. de Barros Neto ${ }^{(1)}$, Osvaldo C.Filho ${ }^{(1)}$, Edson L. Foletto ${ }^{(2)}$ \\ (1) Universidad Federal de Río Grande do Norte, Programa de Post-Graduación en Ingeniería Química, \\ Avenida Salgado Filho 3.000, Lagoa Nova - 59078-970 - Natal - RN - Brasil \\ (e-mail alessandro_alisson@yahoo.com.br) \\ (2) Departamento de ingeniería química, Universidad Federal de Santa Maria, Santa Maria-RS-Brasil
}

* autor a quién debe ser dirigida la correspondencia

Recibido Ago. 26, 2013; Aceptado Oct. 9, 2013; Versión final recibida Dic. 1, 2013

\section{Resumen}

Se ha determinado los puntos de nube de distintos surfactantes polietoxilados y se han caliculado de parámetros termodinámicos como entalpía y entropía de la mezcla utilizando el modelo de Flory-Huggins. Los surfactantes no iónicos son compuestos que pueden ser utilizados como promotores de separación en procesos tales como extracción. Este proceso de separación es basado en un fenómeno químico que ocurre cuando una solución de surfactantes no iónico es sometida al calentamiento, aumentando la temperatura hasta quedar turbia. La temperatura en este momento se denominada punto de nube. La metodología experimental consideró tanto la preparación de soluciones de 0.25 hasta $20 \%$ en masa para cada surfactante estudiado y el calentamiento hasta alcanzar el punto de nube. Los parámetros en el modelo de Flory-Huggins fueron obtenidos usando el método de Levenberg-Marquardt y presentan un ajuste satisfactorio a los datos experimentales para todos los surfactantes estudiados en este trabajo.

\section{Determination of the Parameters of the Flory-Huggins Model to estimate the Cloud Point of Nonionic Surfactants}

\begin{abstract}
The cloud point of different polyethoxylated surfactants have been experimentally determined and thermodynamic parameters such as enthalpy and entropy of mixing using the Flory-Huggins model have been determined. The non-ionic surfactants are compounds that can be used as separation aid in processes such as extraction. This separation process is based on a chemical phenomenon that occurs when a solution of non-ionic surfactants is subjected to warming, increasing the temperature until getting a turbid liquid. The temperature at this point is known as the cloud point. The experimental methodology considered the preparation of solutions of 0.25 to $20 \%$ by mass for each surfactant studied and the heating process until reaching the cloud point. The parameters in the Flory-Huggins model were obtained using the Levenberg-Marquardt method and presented a satisfactory adjustment to the experimental data for all the surfactants studied in this work.
\end{abstract}

Keywords: Levenberg-Marquardt, nonionic surfactants, cloud point, degree of ethoxylation, Flory-Huggins model 


\section{INTRODUCCIÓN}

Los surfactantes son sustancias que, por su estructura química y propiedades, se adsorben en las interfaces liquido-liquido, liquido-gas y solido-liquido, reduciendo la tensión interracial. Se presentan como moléculas antivíricas, o sea, moléculas que poseen en su estructura dos solubilidades distintas asociadas. Estas sustancias poseen, en la misma molécula, grupos polares (hidrofilacios), con afinidad por el agua, y grupos apolares (hidrológicos) con afinidad por los compuestos orgánicos (entre ellos, el aceite) (Formatriz, et., 2005). Los surfactantes no iónicos utilizados en este trabajo son constituidos por sustancias cuyas moléculas, en solución acuosa, no se ionizan.

La solubilidad de estos surfactantes en agua se debe a la presencia de grupos funcionales epoxi que poseen fuerte afinidad por el agua. Los surfactantes no iónicos poseen una característica que cuando calentados forman puntos de nube. Las soluciones de estos surfactantes se turban cuando calentados, formando dos fases a una determinada temperatura y concentración. Esto disminuye la solubilidad del surfactantes en la solución, evidenciando cada vez más la nube (Hombre et al., 2004). El mínimo de curva relacionada por la temperatura en función de la concentración es llamada punto de nube, punto crítico de separación de fases, una con alta concentración de surfactantes llamada fase rica en tenso activo, y otra con baja concentración de surfactantes, llamada de fase diluida. Esta separación en dos fases es, normalmente, acompañada por un aumento de nube de la solución, que puede ser observada visualmente. En la fase diluida, la concentración del surfactantes es próxima a la de la concentración micela crítica (p.m.) (Mu y Zhang, 2000). (Ai-Ghamdi y Nasr-El-Din 1997) investigaron el efecto del punto de nube de surfactantes no iónicos de la familia del Triron-X, (octilfenolpolietoxilado), y observaron que cuanto mayor el grado de etoxilación del tensoactivo mayor es su punto de nube. La determinación del ponto de nube puede ser realizada por potenciometría (Li y Tamura 2010; Fischer y Franzreb 2011) y por el método visual (JingLiang et al., 2009; Inoue y Iwasaki 2010; Didi et al., 2011). Sin embargo, trabajos abordando el estudio del punto de nube y la determinación de los parámetros del punto de nube de surfactantes nonilfenolpolietoxilados y alcohol láurico polietoxilados son escasos en la literatura.

En este trabajo fueron determinados los puntos de nube de varios surfactantes no iónicos tales como nonilfenol-polietoxilados y alcohol láurico polietoxilados. El método Levenberg-Marquardt fue aplicado para la estimación de los parámetros. Fue desarrollada una rutina de puntos de nube de los surfactantes usando el modelo de Flory-Huggins tales como la entalpía de la mezcla $\left(\mathrm{H}_{12}\right)$, entropía de la mezcla $\left(\mathrm{S}_{12}\right)$ y el número de agregaciones del tensoactivo $(\mathrm{N})$. La representación del equilibrio de los tensoactivos no iónicos en medio acuoso a través de la determinación de parámetros como entalpía y entropía son esenciales en la aplicación de los mismos en los procesos industriales, principalmente aquellos que envuelven la extracción de compuestos orgánicos e inorgánicos en medio acuoso (Giokasa et al.,2012;Sato et al.,2013;Suna et al.,2013; Xua et al.,2013), donde la formación de la fase coacervado funciona como una fase extracto, substituyendo así los solventes generalmente tóxicos y agresivos al medio ambiente.

\section{MÉTODO LEVENBERG-MARQUARDT}

El método Levenberg-Marquardt es un método numérico de optimización que consiste en minimizar una función objetivo, a través del ajuste de parámetros del modelo propuesto. (He y Ma 2010) describieron el método numérico Levenberg-Marquardt como muy eficiente para resolver ecuaciones no lineares. $O$ sea, podemos decir que este método es empleado para estimar parámetros de ecuación no lineares (Watson, 2007; Fan y Pan, 2009). De esa manera, se desea ajustar un modelo que represente una determinada curva de solubilidad que es descrita por el punto de nube. Para eso, es necesario determinar parámetros del modelo que pueda ajustar el comportamiento de la curva. Podemos optimizar parámetros $\beta$ de la curva modelo $f\left(x_{i}, \beta\right)$ de modo que la suma de los cuadrados de los desvíos sea mínimo, representado por la siguiente Ecuación (1):

$$
S(\beta)=\sum_{i=1}^{m}\left[y_{i}-f\left(x_{i}, \beta\right)\right]^{2}
$$

El algoritmo Levenberg-Marquardt se basa en un proceso interactivo. Para iniciar una minimización, el usuario debe proporcionar estimativas iniciales para el vector parámetro $\beta$. Para cada iteración, el vector es sustituido por una nueva estimativa $\beta+\delta$. Para determinar $\delta$, las funciones $f\left(x_{i}, \beta+\delta\right)$ son aproximadas por una linearización (ecuación 2):

$$
f\left(x_{i}, \beta+\delta\right) \approx f\left(x_{i}, \beta\right)+j_{i} \delta
$$


Donde:

$j_{i}=\frac{\partial f\left(x_{i}, \beta\right)}{\partial \beta}$

Es el gradiente vector de línea en este caso de $f$ en relación a $\beta$.

Basándose en la suma de los mínimos cuadrados de la función $S(\beta)$ (Ecuación. 1), podemos representar esa suma para una variación $\delta$, o sea, una función $f\left(x_{i}, \beta+\delta\right)$ por la siguiente ecuación:

$S(\beta+\delta) \approx \sum_{i=1}^{m}\left(y_{i}-f\left(x_{i}, \beta\right)-j_{i} \delta\right)^{2}$

Transformando la ecuación 4 en la forma vectorial, resulta:

$S(\beta+\delta) \approx\|y-F(\beta)-J \delta\|^{2}$

Desarrollando la derivada en relación a $\delta$ y estableciendo el resultado igual a cero, resulta:

$\left(J^{T} J\right) \delta=J^{T}[y-F(\beta)]$

Donde, $J^{\top}$ es la matriz Jacobiana, y " $y$ ” y $F$ son vectores.

Levenberg sustituye esa ecuación 6 por una versión amortiguada (Ecuación 7):

$\left(J^{T} J+\lambda I\right) \delta=J^{T}[y-F(\beta)]$

Donde $\lambda$ es el factor de amortecimiento, que es ajustado a cada interacción.

Para mejorar la rapidez del cálculo, Marquardt sustituyó la matriz identidad I por la matriz diagonal, que es representada por los elementos de $J^{T} J$, originando el algoritmo Levenberg-Marquardt (ecuación 8).

$\left(J^{T} J+\lambda \cdot \operatorname{Diag}\left(J^{T} J\right)\right) \delta=J^{T}[y-F(\beta)]$

\section{MODELO DE FLORY-HUGGINS}

El modelo de Flory-Huggins es comúnmente empleado en soluciones poliméricas (Saeki,1997), donde el mismo se basa en las diferencias de los potenciales químicos de las faces (Ecuación 9). Inoe et al. (2003) calcularon los siguientes parámetros a partir de la mezcla binaria de surfactantes: entalpía y entropía de la mezcla bien como el número de agregados de la molécula, representados por $\mathrm{H}_{12}, \mathrm{~S}_{12}$ y $\mathrm{N}$, respectivamente.

$\mu_{1}^{m}-\mu_{1}^{d}=\frac{\partial \Delta G_{m}}{\partial n_{1}}$

Donde $\mu_{1}^{m}$ y $\mu_{1}^{d}$ representan el potencial químico de la fase más concentrada y diluida en relación al componente 1 , respectivamente.

El potencial químico de la fase más concentrada es estimado por la ecuación 10.

$\mu_{1}^{m}=\mu_{1}^{d}+\frac{R T}{\beta}\left[\ln \left(1-\phi_{m}\right)+\left(1-\frac{1}{N}\right) \phi_{m}-\frac{\omega_{12}}{R T} \phi_{m}^{2}\right]$

Donde $\omega_{12}$ es el parámetro de interacción entre las moléculas de tensoactivo y agua el cual es representado por la ecuación 11, y $\beta$ corresponde al número de moléculas del agua.

$\omega_{12}=H_{12}-T S_{12}$

Sustituyendo la ecuación 11 en la 10, resulta en la ecuación 12. 


$$
\frac{\partial \Delta G_{m}}{\partial n_{1}}=\frac{R T}{\beta}\left[\ln \left(1-\phi_{m}\right)+\left(1-\frac{1}{N}\right) \phi_{m}-\frac{H_{12} \phi_{m}^{2}}{R T}+\frac{S_{12} \phi_{m}^{2}}{R}\right]
$$

En el equilibrio, el potencial químico para el componente 1 en la fase diluida es igual al potencial químico en la fase más concentrada $\mu_{1}^{d}=\mu_{1}^{m}$. De esa forma, como el potencial químico es constante, la derivada de la energía libre de Gibbs es igual a cero. Después resulta en la ecuación 13.

$$
T_{\text {cloud }}=\frac{H_{12} \phi_{m}^{2}}{R\left[\ln \left(1-\phi_{m}\right)+\left(1-\frac{1}{N}\right) \phi_{m}\right]+S_{12} \phi_{m}^{2}}
$$

Donde $\mathrm{H}_{12}, \mathrm{~S}_{12}, \mathrm{~N}, \phi_{m}$ son la entalpía entre el surfactantes y el agua, la entropía de la interacción entre el surfactantes y el agua, el número de agregaciones del surfactantes y la fracción volumétrica del soluto, respectivamente.

\section{ESTRUCTURA QUIMICA}

Los surfactantes estudiados presentan las siguientes estructuras moleculares:

- Nonilfenol-polietoxilado:

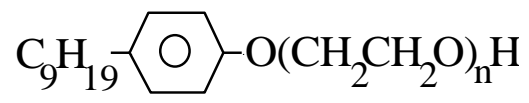

9,5-10 EO: Nonilfenol-polietoxilado $(n=9,5-13)$

- Alcoholes Láuricos polietoxilado:

R-O- $\left(\mathrm{CH}_{2} \mathrm{CH}_{2} \mathrm{O}\right)_{n} \mathrm{H}$

6-9 EO: $\quad$ Alcoholes Láuricos Polietoxilado $(n=6-9)$

\section{MATERIALES Y METODOS}

En este trabajo fueron utilizadas cinco especies de surfactantes del grupo nonilfenol-polietoxilados, variando su grado de etoxilación: 9,5; 10; 11; 12 y 13; y cuatro especies del grupo alcohol láurico polietoxilado, con sus respectivos grados de etoxilaciones: $6,7,8$ y 9 . Estos surfactantes fueron provistos por la empresa Oxiteno (São Paulo, Brasil). Para la preparación de las soluciones, se utilizó agua purificada y deionizada.

La metodología experimental constituyó en la preparación de soluciones sintéticas en la faja de 0.25-20\% en masa de surfactantes (Santos-Ebinuma et al.,2013). Posteriormente, estas soluciones fueron homogeneizadas en agitadores magnéticos. En seguida, fueron colocadas en una célula termostatizada y sometidas a agitación a través de una barra magnética para mantener la solución homogeneizada. Ese aparato experimental fue conectado a un baño termostático. A lo largo de los experimentos, la temperatura del baño fue aumentada de manera gradual al paso que la temperatura de nube de la solución fue verificada visualmente (Taechangam et al 2009;Batıgoç y Akbas 2011). La temperatura del punto de nube fue controlada a través de un termopar (Salvterm 700K- SALCAS). El punto de nube fue establecido a través de las medidas de las temperaturas durante el proceso de calentamiento y resfriamiento de la solución (Wang et al., 2008; Schrader et al.,2013). Para efecto de confirmación del punto de nube, las soluciones fueron resfriadas hasta que se tornasen límpidas (Wang y Feng 2010) nuevamente y después fueron calentadas hasta alcanzar la temperatura de nube con el propósito de confirmación. Los experimentos fueron hechos en triplicado, con reproducibilidad de punto de nube de $\pm 0,1^{\circ} \mathrm{C}$.

\section{OBTENCIÓN DE LOS PARÁMETROS DE PUNTO DE NUBE}

Los parámetros del punto de nube $\mathrm{H}_{12}, \mathrm{~S}_{12}$ e $\mathrm{N}$ fueron calculados utilizando una rutina de minimización aplicando el método numérico Levenberg-Marquardt a través del software Scilab 5.4.1®. El modelo utilizado para estimación de los parámetros del punto de nube es representado por la ecuación 13. La función 
objetivo utilizada para el cálculo de la minimización es representada por la ecuación 14 (Stragevitch y Dávila, 1997).La figura 1 muestra el procedimiento de cálculo para estimación de los parámetros.

$F . O=\sum_{i=1}^{n}\left(\frac{T_{i}^{\mathrm{exp}}-T_{i}^{\text {calc }}}{n_{\text {datos }}}\right)^{2}$

En esta ecuación, $n_{\text {datos }}$ representa el número de datos.

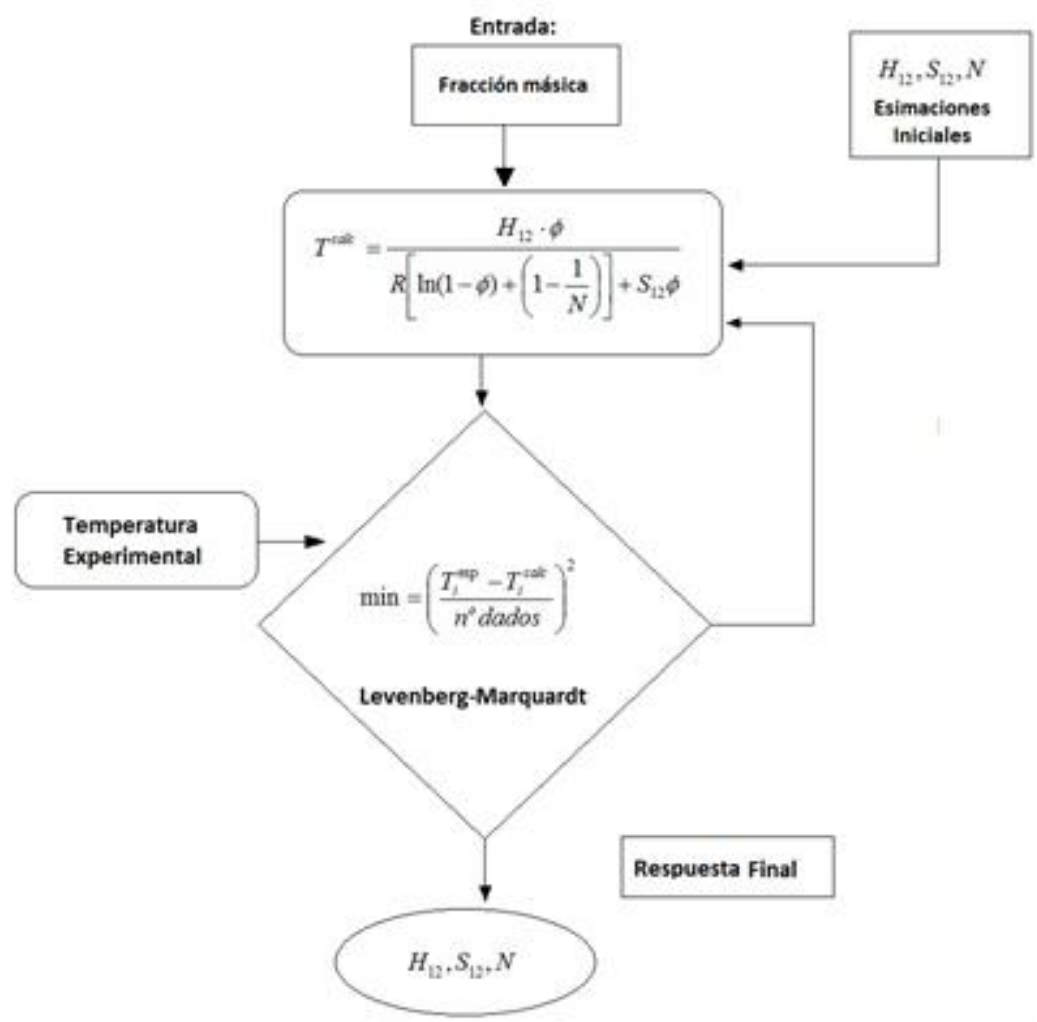

Fig. 1: Método de cálculo para determinación de los parámetros del modelo de Flory-Huggins no Scilab ${ }^{\circledR}$.

\section{RESULTADOS Y DISCUSIÓN}

La figura 2 muestra visualmente el punto de nube formado. Del lado izquierdo, la solución límpida, la cual caracteriza una temperatura abajo del punto de nube, y del lado derecho, una solución con aspecto turbio, en la cual la temperatura de punto de nube fue alcanzada. Las soluciones de estos surfactantes se turban cuando son calentadas, formando dos fases a una determinada temperatura y concentración. Esto disminuye la solubilidad del tensoactivo en la solución, evidenciando cada vez más la nube.

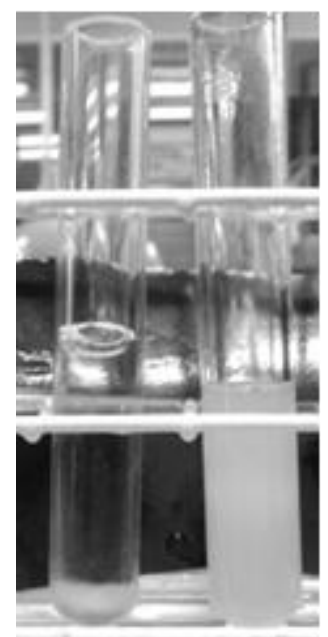

Fig. 2: Representación visual en la formación del punto de nube. 
Ese fenómeno ocurre debido a un movimiento interno de las moléculas de agua que puede vencer las fuerzas de atracción entre la molécula de agua y del surfactante, disminuyendo la solvatación de la parte etoxilada y, consecuentemente, reduciendo a su solubilidad. Eso origina una solubilidad opuesta conocida como cloud point (Inoue et al., 2003).

La figura 3 representa los datos experimentales de temperatura como una función de la concentración del surfactantes bien como el modelo de Flory-Huggins para los nonilfenol-polietoxilados (figura 3a) y los alcoholes láuricos polietoxilados (figura $3 b$ ). A partir de las figuras $3 a$ y $3 b$, es observado un mínimo de la curva de la temperatura en función de la concentración del surfactante, y ese mínimo es llamado de punto de nube o punto crítico de separación de fases (Inoue y Misono, 2008). Eso ocurre porque, arriba del punto de nube, se da la formación de dos fases, una con alta concentración de surfactantes, llamada de coacervado, y otra con baja concentración de surfactantes, llamada fase diluida. Un comportamiento similar también fue observado por otros investigadores (Watanabe y Tanaka, 1978; Clark et al., 2012; Stephen et al., 1992).

De la figura 3, se observa también que la temperatura del punto de nube crece con el aumento de grado de etoxilación, para ambos los tipos de surfactantes. Eso ocurre debido a mayor afinidad de los surfactantes de mayor grado de etoxilación por el agua. O sea, una cadena mayor de unidades de polietoxietilencia interacciona con un número mayor de moléculas de agua. De esta manera, es necesaria una mayor energía para quebrar esas ligaciones y, consecuentemente, la temperatura de nube aumenta. Para ambos los tipos de surfactantes, es observado también que el aumento de la concentración de surfactantes ocasiona un aumento del punto de nube, corroborando los resultados por (Zana y Weill 1985). La tabla 1 muestra los parámetros del punto de nube y los desvíos padrones obtenidos para cada surfactante estudiado.
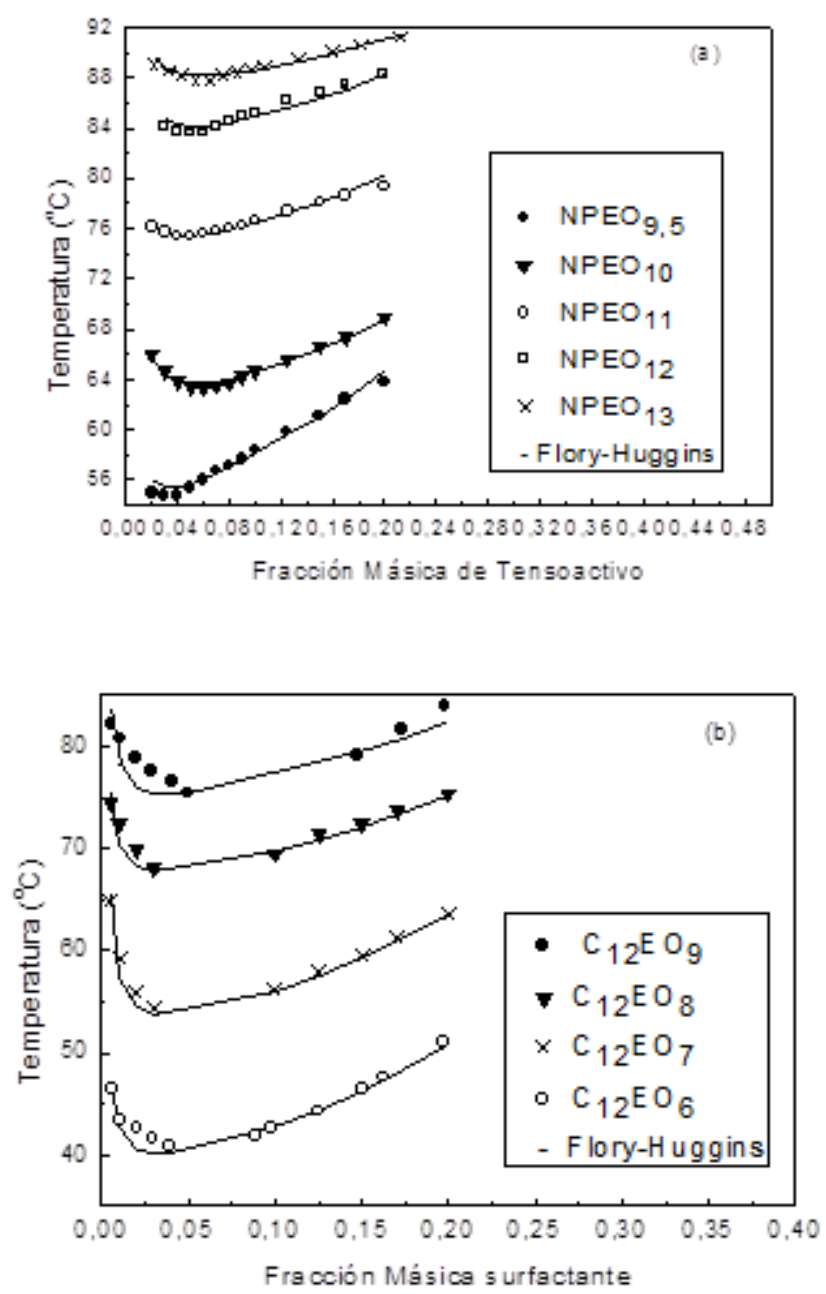

Fig. 3: Representación del modelo de Flory-Huggins para los (a) nonilfenolpolietoxilados y (b) alcoholes láuricos polietoxilados. 
Tabla 1. Parámetros del punto de nube

\begin{tabular}{|c|c|c|c|c|}
\hline Surfactante & $\mathrm{H}_{12}(\mathrm{~J} / \mathrm{mol})$ & $\mathrm{S}_{12}(\mathrm{~J} / \mathrm{mol} . \mathrm{K})$ & $\mathrm{N}$ & Desvío padrón \\
\hline NPEO9.5 & 5,800 & 22 & 2,500 & 0.4896 \\
\hline NPEO10 & 8,600 & 30 & 1,000 & 0.2167 \\
\hline NPEO11 & 11,000 & 36 & 1,500 & 0.2724 \\
\hline NPEO12 & 12,000 & 38 & 1,000 & 0.4029 \\
\hline NPEO13 & 16.800 & 51 & 800 & 0.2847 \\
\hline C12EO6 & 92.3 & 6.64 & 2,755 & 0.7380 \\
\hline C12EO7 & 163.5 & 7.41 & 2,215 & 0.7681 \\
\hline C12EO8 & 323.3 & 9.13 & 2,445 & 0.8617 \\
\hline C12EO9 & 410.4 & 9.81 & 2,065 & 0.7663 \\
\hline
\end{tabular}

Para los surfactantes NPEOn y $\mathrm{C}_{12} E O$ n, se observa que el $\mathrm{H}_{12}$ y $\mathrm{S}_{12}$ crecen con el aumento del grado de etoxilación. Surfactantes con mayor grado de etoxilación necesitan de mayor energía una cantidad de calor (entalpía) para quebrar las ligaciones formadas entre el surfactantes y el agua. Con relación a la entalpía, cuando se aumenta la cantidad de calor (entalpía) las moléculas se agitarán más, probando el aumento de entropía. Por otro lado, el $\mathrm{N}$ disminuye con el aumento del grado de etoxilación para eses surfactantes. Surfactantes con mayor grado de etoxilación poseen un tamaño mayor en su parte polar y con el aumento del diámetro de la parte polar, menos moléculas de surfactantes irán a unirse para formar agregados micelares (Holmberg et al., 2004).

La figura 4 muestra la dependencia del $\mathrm{H}_{12}$ y $\mathrm{S}_{12}$ en relación al grado de etoxilación del tensoactivo NPEOn. Tanto $\mathrm{H}_{12}$ (Fig. 4a) como $\mathrm{S}_{12}$ (Fig. 4b) casi linealmente con el aumento del grado de etoxilación.
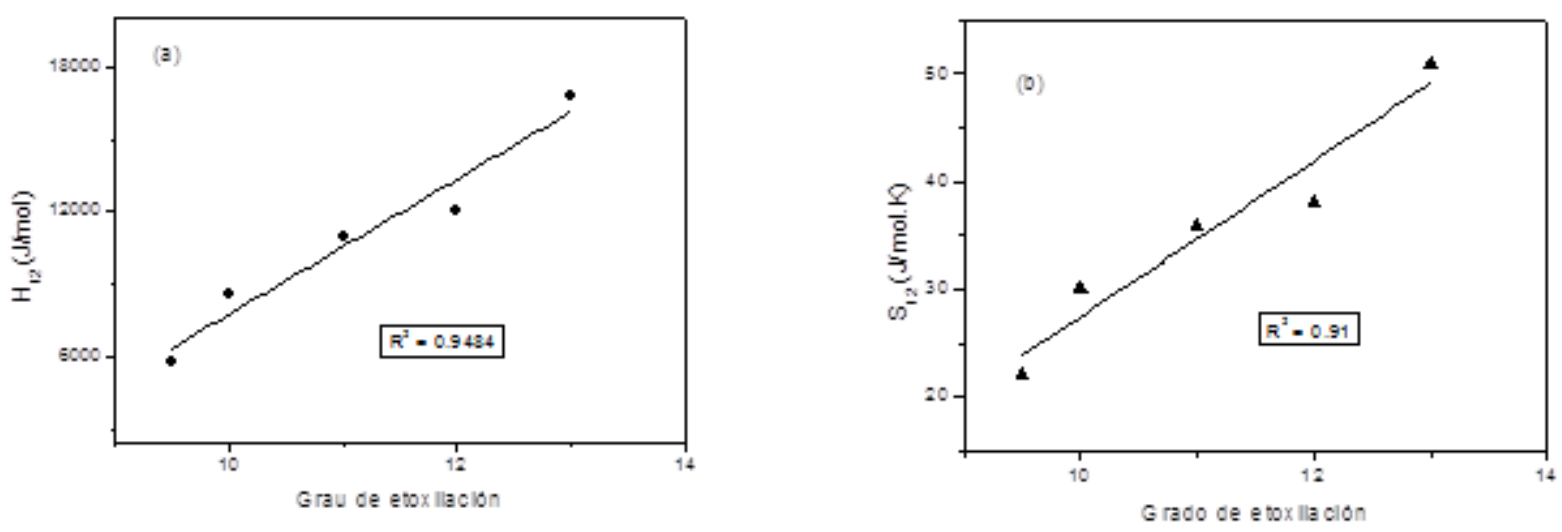

Fig. 4: Representación del parámetro (a) $\mathrm{H}_{12}$ e (b) $\mathrm{S}_{12}$ en función del grado de etoxilación para NPEOn.

Según (Inoue et al., 2003) existe una dependencia de los parámetros $\mathrm{H}_{12}$ y $\mathrm{S}_{12}$ en función del grado de etoxilación. Siendo así, las relaciones lineares para $\mathrm{H}_{12}$ y $\mathrm{S}_{12}$ para los nonilfenol-polietoxilados en función de su grado de etoxilación (n) pueden ser expresadas por las siguientes ecuaciones: Las líneas en las imágenes $4 a$ y $4 b$ fueron hechas de acuerdo con las ecuaciones 15 y 16.

$H_{12}=2790.2 n-20131.7$

$S_{12}=7.23 n-44.87$

La figura 5 muestra la dependencia de $\mathrm{H}_{12}$ y $\mathrm{S}_{12}$ en el grado de etoxilación hacer tensoactivo C12EOn. Ambos $\mathrm{H}_{12}$ y $\mathrm{S}_{12}$ (figura 5) aumentan casi linealmente con el grado de etoxilación. 

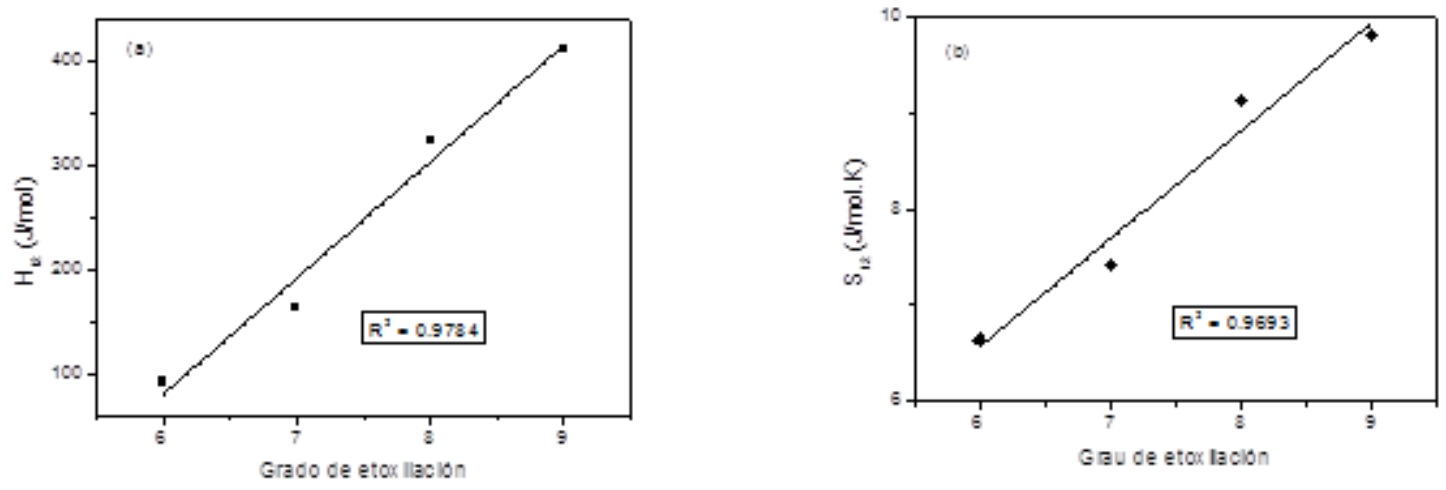

Fig. 5: Representación del parámetro (a) $\mathrm{H}_{12}$ y (b) $\mathrm{S}_{12}$ en función del grado de etoxiliación para C12EOn.

Como relaciones lineares para $\mathrm{H}_{12}$ y $\mathrm{S}_{12}$ para los alcoholes láuricos polietoxilados la función es hacer su grado de etoxiliación (n) que puede ser expresada por las ecuaciones (17) y (18). Las líneas en las imágenes $5 \mathrm{a}$ y $5 \mathrm{~b}$ fueron hechas de acuerdo con las ecuaciones 17 y 18.

$$
H_{12}=111.4 n-588.1
$$

$S_{12}=1.12 n-0.182$

Se observa que la entalpía $H_{12}$ y la entropía $S_{12}$ crece con el aumento del grado de etoxilación para ambos los surfactantes (figura 4 y 5), pues cuando se aumenta el grado de etoxilación más energía es necesaria para quebrar las interacciones entre las moléculas de agua y el tensoactivo y, así, formar el punto de nube (Inoue et at., 2009)

\section{CONCLUSIONES}

Los parámetros del punto de nube determinados a partir del modelo de Flory-Huggins se ajustaron de manera satisfactoria a los datos experimentales para los surfactantes $\mathrm{C}_{12}$ EOn y NPEOn. Los parámetros $\mathrm{H}_{12}$ y $\mathrm{S}_{12}$ del modelo de Flory-Huggins presentaron una dependencia linear con la etoxilación lo que permite dentro de este modelo calcular estos parámetros para otros tensoactivos con etoxilaciones no estudiadas en el trabajo. Sobre el número de agregación los valores obtenidos mostraron una tendencia de reducción con la etoxilación, pero no para todos los tensoactivos y merece estudios con más tensoactivos. De esta forma, estos parámetros tendrán una fundamental importancia en la predicción del punto de nube de los surfactantes polietoxilados, contribuyendo para el desarrollo de futuros trabajos que visan la extracción utilizando punto de nube.

Tabla 2: Datos experimentales y calculados por el modelo de Flory-Huggins para el nonilfenol-polietoxilados

\begin{tabular}{|c|c|c|c|c|c|c|c|c|c|c|c|c|c|c|}
\hline $\begin{array}{l}\text { Fracción } \\
\text { NPEO9.5 }\end{array}$ & $T_{\exp }$ & $T_{\text {cal }}$ & $\begin{array}{l}\text { Fracción } \\
\text { NPE010 }\end{array}$ & $T_{\text {exp }}$ & $\mathrm{T}_{\text {cal }}$ & $\begin{array}{l}\text { Fracción } \\
\text { NPE011 }\end{array}$ & $T_{\exp }$ & $\mathrm{T}_{\text {cal }}$ & \begin{tabular}{|l|l} 
Fracción \\
NPEO12 \\
\end{tabular} & $T_{\exp }$ & $\mathrm{T}_{\text {cal }}$ & $\begin{array}{l}\text { Fracción } \\
\text { NPE013 }\end{array}$ & $T_{\exp }$ & $\mathrm{T}_{\text {cal }}$ \\
\hline 0.02 & 55.0 & 56.0 & 0.02 & 66.0 & 65.8 & 0.02 & 76.1 & 76.3 & 0.03 & 84.1 & 84.7 & 0.02 & 89.0 & 89.7 \\
\hline 0.03 & 54.8 & 55.5 & 0.03 & 64.7 & 64.4 & 0.03 & 75.7 & 75.6 & 0.04 & 83.8 & 84.2 & 0.03 & 88.5 & 88.7 \\
\hline 0.04 & 54.8 & 55.5 & 0.04 & 63.8 & 63.8 & 0.04 & 75.4 & 75.4 & 0.05 & 83.6 & 84.1 & 0.04 & 88.1 & 88.3 \\
\hline 0.05 & 55.4 & 55.8 & 0.05 & 63.4 & 63.7 & 0.05 & 75.4 & 75.4 & 0.06 & 83.6 & 84.1 & 0.05 & 87.8 & 88.2 \\
\hline 0.06 & 56.0 & 56.1 & 0.06 & 63.4 & 63.7 & 0.06 & 75.6 & 75.5 & 0.07 & 84.1 & 84.3 & 0.06 & 87.8 & 88.2 \\
\hline 0.07 & 56.7 & 56.6 & 0.07 & 63.5 & 63.8 & 0.07 & 75.8 & 75.7 & 0.08 & 84.5 & 84.4 & 0.08 & 88.1 & 88.3 \\
\hline 0.08 & 57.2 & 57.0 & 0.08 & 63.7 & 64.1 & 0.08 & 76.0 & 75.9 & 0.09 & 84.9 & 84.6 & 0.09 & 88.4 & 88.4 \\
\hline 0.09 & 57.7 & 57.6 & 0.09 & 64.2 & 64.3 & 0.09 & 76.3 & 76.2 & 0.10 & 85.2 & 84.9 & 0.10 & 88.7 & 88.6 \\
\hline 0.10 & 58.4 & 58.1 & 0.10 & 64.7 & 64.6 & 0.10 & 76.6 & 76.5 & 0.13 & 86.2 & 85.6 & 0.11 & 88.9 & 88.7 \\
\hline 0.13 & 59.8 & 59.5 & 0.13 & 65.6 & 65.5 & 0.13 & 77.4 & 77.3 & 0.15 & 86.9 & 86.4 & 0.13 & 89.5 & 89.3 \\
\hline 0.15 & 61.1 & 61.1 & 0.15 & 66.6 & 66.5 & 0.15 & 78.1 & 78.2 & 0.17 & 87.4 & 87.1 & 0.16 & 90.1 & 89.9 \\
\hline 0.17 & 62.4 & 62.4 & 0.17 & 67.4 & 67.4 & 0.17 & 78.6 & 79.0 & 0.20 & 88.3 & 88.3 & 0.18 & 90.6 & 90.5 \\
\hline 0.20 & 63.8 & 64.6 & 0.20 & 68.9 & 68.8 & 0.20 & 79.3 & 80.2 & & & & 0.21 & 91.2 & 91.3 \\
\hline
\end{tabular}


Tabla 3: Datos experimentales y calculados por el modelo de Flory-Huggins para los alcoholes Laurílicos Polietoxilados

\begin{tabular}{|c|c|c|c|c|c|c|c|c|c|c|c|}
\hline $\begin{array}{c}\text { Fracción } \\
\text { C12EO9 }\end{array}$ & Texp & Tcal & $\begin{array}{c}\text { Fracción } \\
\text { C12EO8 }\end{array}$ & Texp & Tcal & $\begin{array}{c}\text { Fracción } \\
\text { C12EO7 }\end{array}$ & Texp & Tcal & $\begin{array}{c}\text { Fracción } \\
\text { C12EO6 }\end{array}$ & Texp & Tcal \\
\hline 0.005 & 82.1 & 83.8 & 0.005 & 74.5 & 75.6 & 0.005 & 64.9 & 65.7 & 0.006 & 46.5 & 47.2 \\
\hline 0.011 & 80.8 & 78.2 & 0.010 & 72.5 & 70.3 & 0.010 & 59.3 & 57.4 & 0.010 & 43.3 & 42.6 \\
\hline 0.020 & 78.9 & 76.0 & 0.020 & 69.9 & 68.2 & 0.020 & 56 & 54.4 & 0.020 & 42.6 & 40.5 \\
\hline 0.029 & 77.5 & 75.5 & 0.030 & 68.1 & 67.8 & 0.030 & 54.3 & 53.8 & 0.029 & 41.5 & 40.2 \\
\hline 0.040 & 76.5 & 75.4 & 0.100 & 69.5 & 69.8 & 0.100 & 56.2 & 56.1 & 0.039 & 40.8 & 40.2 \\
\hline 0.049 & 75.4 & 75.5 & 0.125 & 71.4 & 70.9 & 0.125 & 57.9 & 57.6 & 0.089 & 41.8 & 42.2 \\
\hline 0.148 & 79.1 & 79.4 & 0.150 & 72.5 & 72.2 & 0.150 & 59.5 & 59.3 & 0.098 & 42.7 & 42.8 \\
\hline 0.173 & 81.7 & 80.8 & 0.170 & 73.8 & 73.3 & 0.170 & 61.2 & 60.8 & 0.124 & 44.3 & 44.4 \\
\hline 0.198 & 84 & 82.2 & 0.200 & 75.4 & 75.2 & 0.200 & 63.5 & 63.4 & 0.151 & 46.5 & 46.4 \\
\hline & & & & & & & & & 0.163 & 47.6 & 47.4 \\
\hline & & & & & & & & & 0.197 & 51.1 & 50.6 \\
\hline
\end{tabular}

\section{REFERENCIAS}

Ai-Ghamdi A. M., Nasr-El-Din H.A.; Effect of oilfield chemicals on the cloud point of nonionic. Colloid And Surface A, v.125, p.5-18, (1997).

Batıgoç C.y Akbas H.; Spectrophotometric determination of cloud point of Brij 35 nonionic surfactant, Fluid Phase Equilibria,v.303,p. 91-95, (2011).

Clark E.A., Lipson J.E.G.; LCST and UCST behavior in polymer solutions and blends, Polymer, v. 53, p.536545, (2012).

Didi M. A., Sekkal A. R., Villemin D.; Cloud-point extraction of bismuth (III) with nonionic surfactants in aqueous solutions, Colloids and Surfaces A: Physicochem. Eng. Aspects, v.375,p. 169-177,(2011).

Duarte L. J. N, Canselier J.P.; Extração no ponto nuvem de compostos orgânicos homólogos e isômeros. Química Nova, v.1, p.426-432, (2005).

Fan J., Pan J.; A note on the Levenberg-Marquardt parameter, Applied Mathematics and Computation, v.207, p. 351-359, (2009).

Fischer I. y Franzreb M.; Direct determination of the composition of aqueous micellar two-phase systems (AMTPS) using potentiometric titration-A rapid tool for detergent-based bioseparation, Colloids and Surfaces A: Physicochem. Eng. Aspects,v.377,p. 97-102, (2011).

Formariz T.P., Urban M. C. C., da Silva Júnior A. A., Gremião M. P. D.y de Oliveira A. G.; Microemulsões e fases líquidas cristalinas como sistemas de liberação de fármacos. Revista brasileira de ciências farmacêuticas, v. 41, p. 301-313, (2005).

Giokasa D. L., Zhub Q., Panb Q, Chisvert A.; Cloud point-dispersive -solid phase extraction of hydrophobic organic compounds onto highly hydrophobic core-shell $\mathrm{Fe}_{2} \mathrm{O}_{3} \mathrm{C}$ magnetic nanoparticles, Journal of Chromatography A, v.1251, p. 33-39, (2012).

He C. y Ma C.; A smoothing self-adaptive Levenberg-Marquardt algorithm for solving system of nonlinear inequalities, Applied Mathematics and Computation,v.216,p. 3056-3063, (2010).

Holmberg K., Jonsson B., Kronberg B. y Lindman B.; Surfactants and polymers in aqueous solution, 2ª́Edição, Jonh Wiley \& Sons LTD, 49-54, Chichester, Inglaterra, (2004).

Inoue T. y Iwasaki Y.; Cloud point phenomena of polyoxyethylene-type surfactants in ionic liquid mixtures of emimBF4 and hmimBF4, Journal of Colloid and Interface Science, v.348,p. 522-528,(2010).

Inoue T., Higuchi Y. y Misono T.; Differential scanning calorimetric study of nonionic surfactant mixtures with a room temperature ionic liquid, bmimBF4, Journal of Colloid and Interface Science,v.338,p.308-311,(2009).

Inoue, T., Ohmura, H. y Murata, D.; Cloud point temperature of polyoxyethylene-type nonionic. Colloid And Interface Science, v.258, p.374-382, (2003).

Inoue T. y Misono, T.; Cloud point phenomena for POE-type nonionic surfactant in a model room temperature ionic liquid. Colloid And Interface Science, v.326, p.483-489, (2008). 
Jing-Liang L., Dong-Shun B., Bing-Hung C. Effects of additives on the cloud points of selected nonionic linear ethoxylated alcohol surfactants, Colloids and Surfaces A: Physicochem. Eng. Aspects, v.346, p. 237243 (2009).

Li X. y Tamura K., Cloud point measurements of 1-butyl-2,3-dimethylimidazolium tetrafluoroborate with alcohols, Journal Chemical Thermodynamics,v. 42,p. 1478-1484, (2010).

Maniasso N.; Ambientes micelares em química analítica, Quimica Nova, V. 24, p. 87-93, (2001).

Mu, J.M., Li G. y Zhang W.; Determination of the second CMCs of dodecyl polyoxyethylene polyoxyethylene ether by the methods of cloud point, fluorescence, and viscosity. Colloid And Surface A, 194, .1-6, (2001).

Nilsson P.G., Pacynko W.F. y Tiddy G.J.T.; 'Clouding' in Zwitterionic surfactant and water systems -the influence of additives on the upper consolute loop of the decyldimethylammonioethane sulfate-water system, Current Opinion in Colloid and Interface Science, v. 9, p. 117-123, (2004).

Watanabe H. y Tanaka H.; A non-ionic surfactant as a new solvent for liquid-liquid extraction of zinco (II) with I-(2-pyridylazo)-2-naphthol, Talanta, v.25, p.585-589, (1978).

Wang Z., Xu J., Zhang W., Zhuang B.y Qi H.; Cloud point of nonionic surfactant Triton X-45 in aqueous solution, Colloids and Surfaces B: Biointerfaces, v.61, p. 118-122, (2008).

Watson G.A; A Levenberg-Marquardt method for estimating polygonal regions, Journal of Computational and Applied Mathematics, v.208, p. $331-340$, (2007).

Rybinski W. V.; Alkyl glycosides and polyglycosides, Colloid \& Interface Science, v.1, p.587-597, (1996).

Saeki S.; Calculation of combinatory entropy in complex polymer solutions based on the Flory-Huggins theory, Fluid Phase Equilibria, v.136, p. 79-85, (1997).

Santos-Ebinuma V. C., Lopes A. M.,Converti A.,Júnior A. P., Rangel-Yagui C. O.; Behavior of Triton X-114 cloud point in the presence of inorganic electrolytes, Fluid Phase Equilibria,v. 360,p. 435-438, (2013).

Sato N., Mori M., Itabashi H.; Cloud point extraction of $\mathrm{Cu}$ (II) using a mixture of triton X-100 and dithizone with a salting-out effect and its application to visual determination, Talanta, v.117, p. 376-381, (2013).

Schrader P., Culaguin-Chicaroux A., Enders S.;Phase behavior of the water+nonionic surfactant $\left(\mathrm{C}_{12} \mathrm{E}_{8}\right)+1$ dodecene ternary system across a wide temperature range, Chem. Eng. Science,v.93,p. 131-139, (2013).

Stragevitch L., y Dávila S.G.; Application of a Generalized Maximum Likelihood Method In the Reduction of Multicomponent liquid-liquid equilibrium data, braz. j. chem. Eng. Vol. 14, (1997).

Stephen J. M., Qian C., Eichinger B. E.; Phase diagrams of quasi-binary polymer systems with LCST/UCST spinodals and hour-glass cloud-point curves, Polymer, v.33, p.5105-5108, (1992).

Wang Z. y Feng $\mathrm{H}$.; Double cloud point of ethylene oxide-propylene oxide triblock copolymer in an aqueous solution, Colloids and Surfaces A: Physicochem. Eng. Aspects,v.362,p.110-116,(2010).

Suna M., Liua G., Wu Q.; Speciation of organic and inorganic selenium in selenium-enriched rice by graphite furnace atomic absorption spectrometry after cloudv point extraction, Food Chemistry, v.141, p. 66-71, (2013).

Watanabe $\mathrm{H}$. y Tanaka $\mathrm{H}$; ; A non-ionic surfactant as a new solvent for liquid-liquid extraction of zinc (ii) with I-(2-pyridylazo)-2-naphthol, Talanta, v.25, p.585-589, (1978).

Zana R. y Weill C.; Effect of temperature on the aggregation behavior of nonionic surfactants in aqueous solutions, Journal de Physique, v.46, p.953-960, (1985).

Taechangam P., Scamehorn J. F., Osuwan S., Rirksomboon T; Effect of nonionic surfactant molecular structure on cloud point extraction of phenol from wastewater, Colloids and Surfaces A: Physicochem. Eng. Aspects,v.347,p. 200-209,(2009).

Xua H., Zhanga W., Zhang X., Wanga J., Wanga J.; Simultaneous preconcentration of cobalt, nickel and copper in water samples by cloud point extraction method and their determination by flame atomic absorption spectrometry, Procedia Environmental Sciences, v.18, p. 258 - 263, (2013). 